\title{
DFT study of crystalline nitrosyl compounds
}

\author{
Bernard Delley* \\ Paul Scherrer Institut, WHGA, 5232 Villigen PSI, Switzerland
}

Received August 17, 2007; accepted November 21, 2007

\section{Density functional theory / Nitrosyl compounds / Metastable state / Energy barrier}

\begin{abstract}
Nitrosyl linkage isomer compounds are investigated with density functional theory for realistic crystal structures. As compared to free anion models, significant differences are found for various properties. At the metastable state $\mathrm{S} 1$, a crystal environment contribution of 30$40 \mathrm{~cm}^{-1}$ to the red shift exists. The barrier against thermal decomposition of S1 in SNP is significantly lowered by $300 \mathrm{meV}$.
\end{abstract}

\section{Introduction}

Almost all of the theoretical studies on nitrosyl complexes have been done using gas phase molecular models. Gas phase models have been treated starting with the early semiempirical molecular orbital spectroscopic study of the ground state [1]. Semiempirical investigations of ground state structures with bent NO ligand [2] have followed. An early density functional study (DFT) on sodium nitroprusside $\left(\mathrm{SNP}: \mathrm{Na}_{2}\left[\mathrm{Fe}(\mathrm{CN})_{5} \mathrm{NO}\right] \cdot 2 \mathrm{H}_{2} \mathrm{O}\right)$ [4] took hints at atomic rearrangements from an earlier photocrystallographic study [5] serious and found metastable states as local minima on the energy surface. These calculations showed the local minima to have Mössbauer quadrupole splitting in convincing agreement with experiment for each of the states in question: ground state (GS), metastable states $\mathrm{S} 1$ and $\mathrm{S} 2$. Furthermore vibrational analysis found a significant softening of the NO stretch frequency going from GS to $\mathrm{S} 1$ and even more pronounced softening on going to $\mathrm{S} 2$. This is in semiquantitative agreement with the spectral features emerging with $\mathrm{S} 1$ and $\mathrm{S} 2$ population in infrared and Raman spectroscopy. The calculated relative energies of GS, S1 and S2 are consistent with calorimetric measurements. The existence of high energy barriers between these basins on the energy surface was demonstrated using constrained optimizations for scanning the energy hypersurface and for finding the saddle points. The high barriers separating the metastable states are in qualitative agreement with experimental Arrhenius behavior and the very long lifetime at low temperature. On the basis of a zeroth order calculation of the excited state sur-

\footnotetext{
* e-mail: bernard.delley@psi.ch
}

face, a mechanism for population of the metastable states was proposed. A number of DFT calculations were done later for the same compound as well as for other nitrosyl compounds [7-14]. These calculations have confirmed various aspects of the calculation [4] and found no objections. Notably the time dependent DFT calculation of Gorelsky and Lever [7] gives an advanced treatment to the excited states of ground and metastable states, confirming the general picture that was given on the basis of the zeroth order calculation in 1997. HF calculations give a clearly different orbital ordering and a much larger (unrealistic) excitation energy. A recent multireference configuration interaction calculation scanned the most relevant coordinates of the energy hypersurface while keeping the remaining internal coordinates constant [3]. S1 and S2 were found in excellent near quantitive agreement with DFT. Similar to DFT the S1, S2 minima were found too high in energy with respect to the ground state. The existence of high barriers was confirmed by the multireference calculations. However, barrier height was underestimated as compared to experimentally accepted values.

\section{Methods}

For the DFT calculations the $\mathrm{DMol}^{3}$ method is used [15]. This method allows to study vacuum boundary conditions for gas phase molecules. With the same basis sets and other details of the method kept consistent, also bulk crystal bandstructures and surface slab models can be treated [16]. Recently the COSMO (conductor-like screening model) for a liquid environment has been generalized for infinite polymers, surfaces and internal voids, such as zeolites [17], but, that is not used in the present work. The present calculations are done using the PBE functional [19]. Variational numerical local orbital basis sets at the level of the default DND [15] or alternatively the DNP [15] were used. These numerical basis sets are constructed with an atomic response idea in mind. They were demonstrated to represent well a wide class of systems. DND does not include a $p$-polarization function on the $\mathrm{H}$ atoms, and is thus a moderatly less accurate basis set the DNP. As the $\mathrm{H}$ atoms are somewhat peripheral to our present focus on the metal-NO bonding, only minor influence of the $p$-polarization functions on $\mathrm{H}$ is seen for the properties of interest. The final results presented here are all calcu- 
lated at DNP basis set level. The auxiliary density for transition metal elements, open $d$-shells, includes $L=4$ multipolar components. For other elements the auxiliary density goes up to octupoles except for $\mathrm{H}$, where truncation is at quadrupoles, according to the default setting of $\mathrm{DMol}^{3}$. The earlier calculations of Ref. [4] were truncated at octupoles for the metal according to the default setting of that time. This former stronger truncation has an absolutely minor effect for the compounds of interest here. $k$-point sampling for the crystalline compounds is with a $2 \times 2 \times 2$ mesh containing the $\Gamma$-point. For the supercell used for the dispersion calculations a $1 \times 2 \times 2 k$-points mesh was used.

The vibrations were calculated in harmonic approximation, using numerical differentiation of analytical forces. Phonon dispersion was investigated with a $6 \times 1 \times 1$ supercell, leading a resolution of $6 k$-points across the Brillouin zone for vibrations.

Saddle point search and optimization were done with the method of Govind [18]. Our refinement of the method is efficacious in finding a saddle point between to local minima on the energy hypersurface as well as in precisely locating the saddlepoint. For graphical presentation of the energy barrier, a generalized quadratic synchronous transit path [18] is put through the two local minima and the saddle point. The location along the path shown is based on the root mean square (RMS) measure of coordinate differences:

$$
p=\operatorname{RMS}\left(c_{i}-c_{r}\right) /\left(\operatorname{RMS}\left(c_{i}-c_{r}\right)+\operatorname{RMS}\left(c_{i}-c_{p}\right)\right)
$$

where $c_{i}, c_{r}, c_{p}$ denote the coordinates of the intermediate, reactant side structure and product structure respectively. The RMS operator takes the RMS value of the difference vector. The path parameter $p$ is interpreted as an approximate reaction coordinate. For the double barriers of interest here the partial path parameters for GS-S2 and for $\mathrm{S} 2-\mathrm{S} 1$ are joined into a single picture.

For almost 600 molecules, involving atoms up to element $\mathrm{Cl}$, expectations on the level of agreement with experimental enthalpies have been established [20]. The root mean square deviation of relative energies from experimental ones at the local minima is to be expected to be about $0.2 \mathrm{eV}$ for high quality density functional and wavefunction methods alike. The statistical analysis shows that the DNP basis set used here is providing results of very consistent accuracy for DFT calculations, that is not exceeded substantially with much larger basis sets.

\section{Results}

Already Ref. [4] pointed out the excellent agreement between the experimental and calculated crystal structures for the ground state of sodium nitroprusside (SNP : $\mathrm{Na}_{2}\left[\mathrm{Fe}(\mathrm{CN})_{5} \mathrm{NO}\right] \cdot 2 \mathrm{H}_{2} \mathrm{O}$ ). The lowest energy molecular structure for S2 was found with the FeNO plane containing two of the equatorial ligands. We refer to this structure as eclipsed. The 'staggered' structure with FeNO in the mirror plane connecting pairs of equatorial ligands is a saddle point. The staggered structure is compatible with a non-statistical crystal model with Pnnm symmetry

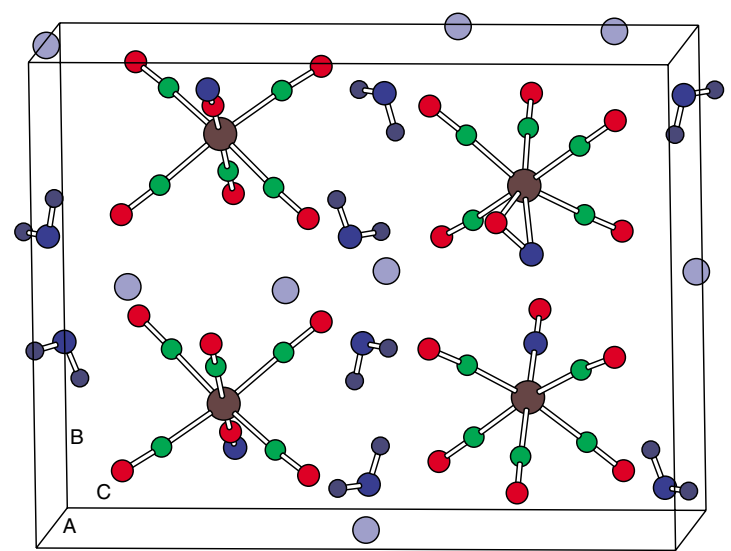

Fig. 1. Crystal model of SNP: (S2 GS S1 GS), the eclipsed S2 (see text) is in the upper right corner, $\mathrm{S} 1$ below.

which was calculated before [4]. The present calculations show that the staggered S2 structure is a saddle point in the solid. These calculations were done with one of the four molecular units of the cell in S2 geometry and the other three in GS geometry. With deformation along the unstable mode as a starting point a low symmetry $P 1$ model was found with approximately eclipsed S2 geometry. The barrier height of staggered versus eclipsed S2 at concentration $1 / 4$ is $94 \mathrm{meV}$. The earlier molecular finding was $84 \mathrm{meV}$. The eclipsed S2 geometry in the crystal was inferred from neutron scattering experiments [22]. For illustration a low symmetry crystal cell for SNP is shown in Fig. 1. with one anion in S2 (eclipsed) conformation, the other anion in the Pnnm mirror plane is in S1 and the remaining two anions are in GS conformation. This model is referred to as S2 GS S1 GS. It is a crystal lattice with $25 \%$ population of $\mathrm{S} 1$ and $\mathrm{S} 2$ each.

\subsection{Vibrational properties}

Vibrational spectra provide a fingerprint of the populated metastable states. A particularly clear picture comes from

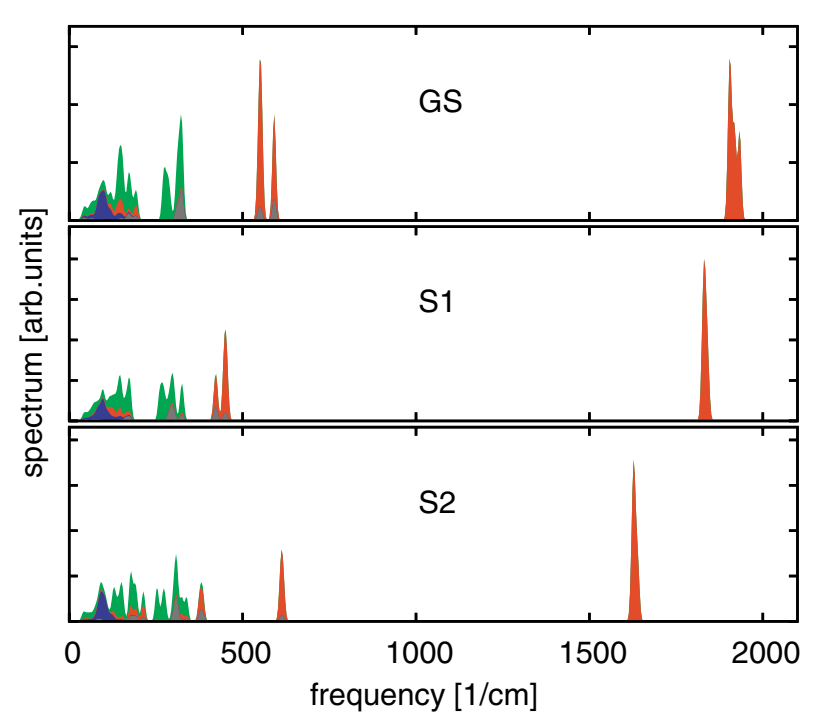

Fig. 2. Vibrational spectrum of $\mathrm{K}_{2}\left[\mathrm{RuCl}_{5} \mathrm{NO}\right]$, red $\left(f>400 \mathrm{~cm}^{-1}\right)$ $\mathrm{NO}$ group, grey $\mathrm{Fe}$, green $\mathrm{Cl}$ and blue $\mathrm{K}$ character of the normal modes (modes are Gaussian broadend for figure $\sigma=5 \mathrm{~cm}^{-1}$ ). 


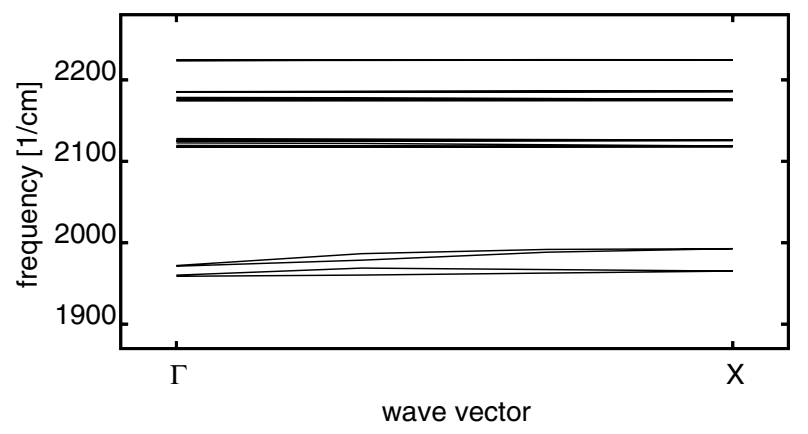

Fig. 3. Phonon dispersion relations in $\mathrm{X}$ direction in the frequency range $1900-2300 \mathrm{~cm}^{-1}$ calculated for ground state SNP.

the spectrum of the most simple $\mathrm{MNO}^{6}$ compound: $\mathrm{K}_{2}\left[\mathrm{RuCl}_{5} \mathrm{NO}\right]$. These calculations with $100 \%$ population retaining full symmetry of GS were done in 2001 and were only presented in talks before. Normal modes components on various groups are represented in color. There is progressive softening of the NO stretch mode on going from GS to S1 and S2, see Fig. 2. In this compound the two FeNO wag modes remain spectrally well separated from backbone modes. There is clear softening of the wag modes from GS to $\mathrm{S} 1$. For $\mathrm{S} 2$ the two wag modes become very different and split by a sizeable amount. This type of calculations has produced $\Gamma$-point phonons. For well isolated molecular entities, as is the case for the class of compounds studied here phonon dispersion should be small. That polarization and long range electrostics dependent effects such as longitudinal to transverse optical phonon splitting remains unimportant, is demonstrated by the present phonon dispersion results, Fig. 3. The dispersion study is done by calculating all $\Gamma$-point vibrations for a six-fold supercell, a cell containing 504 atoms. Wavevector analysis of the vibrations yields the dispersion relations.

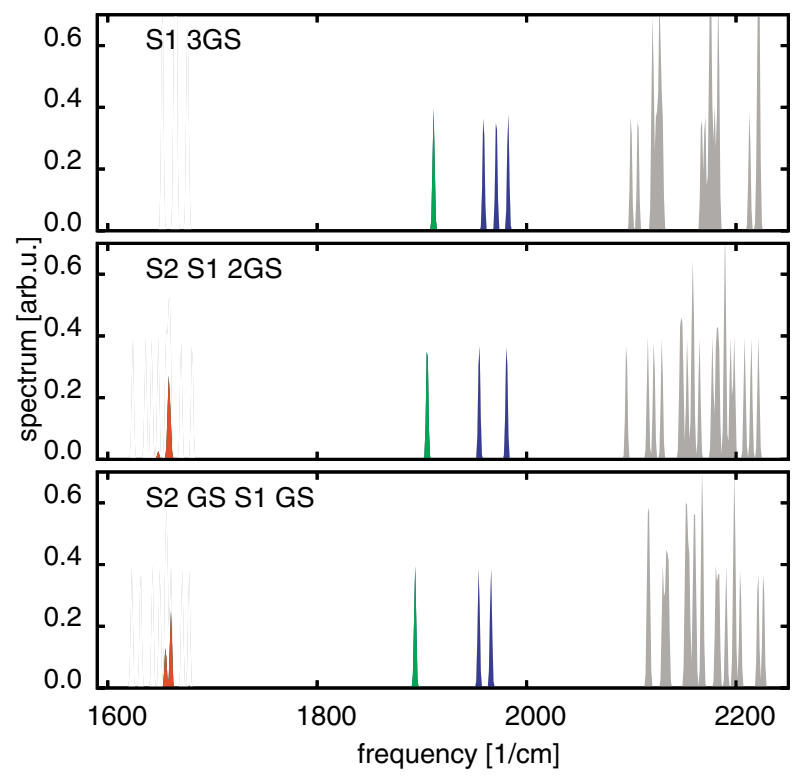

Fig. 4. Vibrational spectrum in the $\mathrm{NO}, \mathrm{CN}$ stretch frequency range. The colors represent partial intensities of the modes on $\mathrm{NO}(\mathrm{GS})$ blue $\left(f \approx 1975 \mathrm{~cm}^{-1}\right), \quad \mathrm{NO}(\mathrm{S} 1)$ green $\left(f \approx 1900 \mathrm{~cm}^{-1}\right), \quad \mathrm{NO}(\mathrm{S} 2)$ red $\left(f \approx 1680 \mathrm{~cm}^{-1}\right)$ and $\mathrm{CN}$ light grey. Vibrational intensity due to crystal water at the lower frequency end of the figure have been removed for clarity.

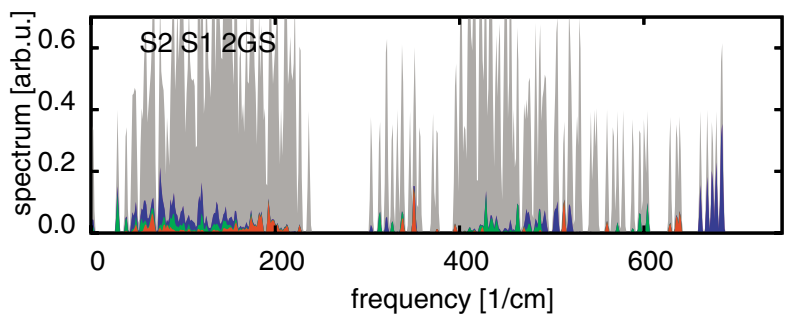

Fig. 5. Vibrational spectrum in the low frequence range. The colors highlight the partial intensities on $\mathrm{NO}(\mathrm{GS})$ blue, $\mathrm{NO}(\mathrm{S} 1)$ green, $\mathrm{NO}(\mathrm{S} 2)$ red and other atoms in light grey.

$\Gamma$-point vibrational calculations have been done for a number of SNP structures, only a selection is shown here. Spectra in the stretch frequency range are shown in Fig. 4. Normal mode components on the various ligands are highlighted in color. As compared to molecular density functional calculations for SNP, the red shift of the NO stretch mode on going to $\mathrm{S} 1$ is much more pronounced than in the molecular anion calculations. This is partly due to the presence of a realistic crystalline environment. For an otherwise identical calculation, the crystal environment increases the redshift by $30-40 \mathrm{~cm}^{-1}$. Moreover, sensitivity of the $\mathrm{CN}$ and $\mathrm{NO}(\mathrm{GS})$ vibrations to details of the crystal environment is suggested by the present results. The still too small red shift of the $\mathrm{NO}(\mathrm{S} 1)$ stretch as compared to experiment is probably a shortcoming common to present density functional approximations. Too small S1 red shifts appear to be widespread. It is clear that the NO metal bonding is quite subtle. Expectations for relative energetics should be seen in perspective with the statistics from Ref. [20]. If the energies at points of the hypersurface cannot be calculated with perfect accuracy, then this must be expected for the curvature too. For the NO stretch the systematic errors of the approximations in electronic structure theory are non-negligible and are only slightly smaller than this $\mathrm{S} 1$ redshift.

Low frequency modes associated with metastable states are mostly drowned in the multitude of backbone vibrations of SNP. However, the present calculated S1 components near $600 \mathrm{~cm}^{-1}$ and the S2 components near 560 and $640 \mathrm{~cm}^{-1}$ (Fig. 5.) may well correspond the enhancement of IR absorption by S1 S2 population observed by Guida [24] with three peaks between 530 and $600 \mathrm{~cm}^{-1}$. The peaks at 566 and $583 \mathrm{~cm}^{-1}$ observed in resonant raman spectra [25] most probably also corresponds to the present $\mathrm{S} 1$ components at $600 \mathrm{~cm}^{-1}$.

\subsection{Energy barriers}

Optimization of the stationary point along the generalized quadratic synchronous transit path [18] yields a saddle point and defines an approximate reaction path along that transit. The saddle points have been checked for the presence of exactly one negative Hessian eigenvalue.

Experimentally, the height of the energy barrier with respect to deexcitation of a metastable state can be determined by Arrhenius analysis of calorimetric measurements. Therefore, the saddle point energies are referred to the relevant metastable state energy minimum, for example $\mathrm{S} 2$ in $\mathrm{TS}(\mathrm{S} 2 \rightarrow \mathrm{GS})$. Calculated and experimental values 
Table 1. SNP energies of metastable states referred to ground state. Barriers for deexcitation from metastable states. Experiment i) isothermal d) dynamic.

\begin{tabular}{lllll}
\hline & $\mathrm{TS}(\mathrm{S} 2 \rightarrow \mathrm{GS})$ & $\mathrm{E}(\mathrm{S} 2)$ & $\mathrm{TS}(\mathrm{S} 1 \rightarrow \mathrm{S} 2)$ & $\mathrm{E}(\mathrm{S} 1)$ \\
\hline anion & 0.75 & 1.43 & 1.18 & 1.63 \\
crystal & 0.79 & 1.36 & 0.88 & 1.61 \\
$\operatorname{exp~i~[21]~}$ & 0.46 & & 0.69 & \\
$\operatorname{exp~d~[21]~}$ & 0.48 & & 0.67 & \\
$\exp *$ & & 1.0 & & 1.1 \\
\hline
\end{tabular}

for metastable state energies of SNP are shown in Table 1. The experimental measurement of the metastable state energy is a challenge, since not only a calorimetric measurement of deexcitation enthalpy but also a measurement of initial population is required. In the case of SNP, the population can be inferred alternatively from Mössbauer spectra and from optical spectra.

The present results clearly show that the crystal environment in SNP significantly lowers the $\mathrm{TS}(\mathrm{S} 1 \rightarrow \mathrm{S} 2)$ barrier as compared to the anion model. This brings this barrier significanly closer to the experimental Arrhenius value. The crystal environment effect remains small for the $\mathrm{TS}(\mathrm{S} 2 \rightarrow \mathrm{GS})$ barrier.

The DFT barriers for SNP are higher that the Arrhenius value. In fact published and unpublished DFT barriers for nitrosyl compounds are systematically somewhat too high, however, within the expectations raised by Ref. [20]. The too high DFT barriers for nitrosyl compounds are opposite to understimated barries for other compounds as reported in [26, 27].

Figure 6 shows a comparison of the barrier profiles along the generalized quadratic transit path for the free anion and the crystal. The energy surface near $\mathrm{TS}(\mathrm{S} 2 \rightarrow \mathrm{GS})$ looks very much textbook like with two parabolic surfaces intersecting. The other barrier $\mathrm{TS}(\mathrm{S} 1 \rightarrow \mathrm{S} 2)$ has a sholder on the S2 side in the case of the anion model. Such shoulders have also been found in other nitrosyl compounds for example in $\left[\mathrm{Ru}\left(\mathrm{NH}_{3}\right)_{4}\left(\mathrm{H}_{2} \mathrm{O}\right) \mathrm{NO}\right]^{3+}$ see Ref. [12] $\left[\mathrm{RuCl}(\mathrm{py})_{4} \mathrm{NO}\right]^{2+}$ Ref. [14]. This shoulder is arising from the HOMO orbital aquiring a $\delta$ bonding character of Fe $d$-with the antibonding $\pi$ NO orbital normal to the FeNO plane. This is illustrated in Fig. 7. for the $\mathrm{RuCl}_{5}$ anion.

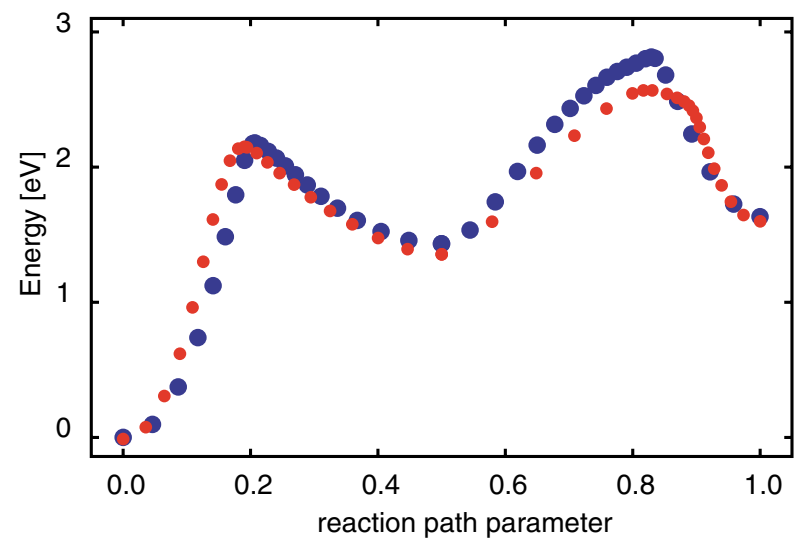

Fig. 6. Calculated energy surface along reaction path, dark-grey anion model, light grey crystal.

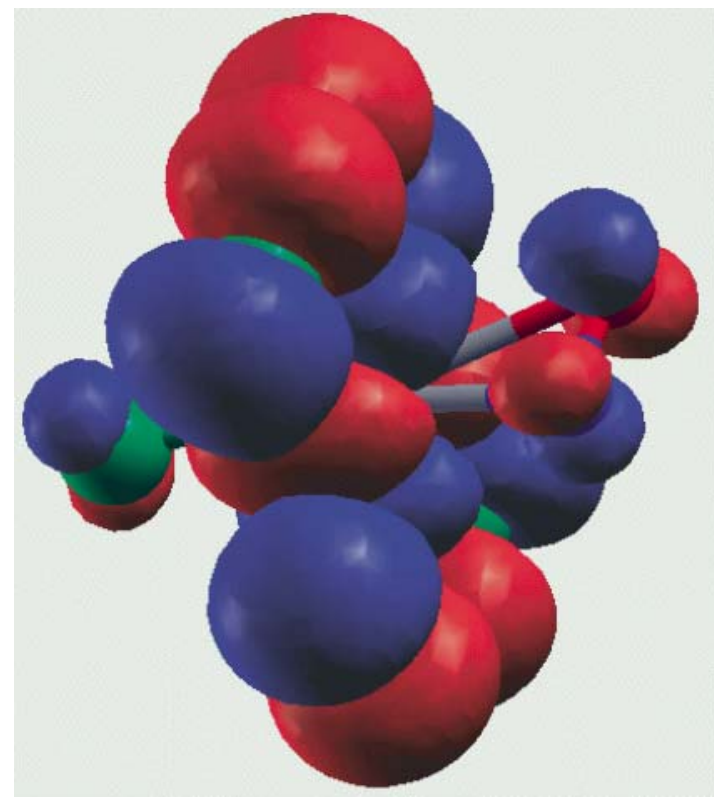

Fig. 7. $\mathrm{HOMO}$ orbital of $\left[\mathrm{RuCl}_{5}\right]^{2+}$ near saddle point $\mathrm{TS}(\mathrm{S} 1 \rightarrow \mathrm{S} 2)$.

\section{Summary and conclusions}

The present calculations have shown that the crystal environment significantly influences the properties of nitrosyl compounds. In particular it was shown that the NO bond of the S1 metastable state gets weakened by the environment and thus its stretch vibrations more red shifted than seen with anion only models. The energy minimum for S2 in crystalline SNP is found to be in the eclised conformation, thus breaking the mirror symmetry of the crystal locally. Furthermore it was shown that the saddle point energies can be significantly lowered due to the crystal. This turns out to be important for the $\mathrm{TS}(\mathrm{S} 1 \rightarrow \mathrm{S} 2)$ of SNP, while the $\mathrm{TS}(\mathrm{S} 2 \rightarrow \mathrm{GS})$ is almost unchanged as compared to the free anion. The calculated $\mathrm{TS}(\mathrm{S} 1 \rightarrow \mathrm{S} 2)$ saddle point is commonly rather broad in nitrosyl compounds. This is explained by the formation of a $\delta$ bond for the HOMO orbital between an Metal $d$ - and the antibonding $\pi$ NO orbital normal the the Metal NO plane.

While crystal effects are important for the detailed properties of nitrosyl linkage isomers, it is also clear that free anion studies go a long way in semi-quantitatively explaining most properties.

Acknowledgments. The author thanks T. Woike, P. Coppens, D. Schaniel and J. Schefer for stimulating discussions and early communication of unpublished results.

\section{References}

[1] Manoharan, P. T.; Gray, H. B.: Electronic structure of the nitroprusside ion. J. Am. Chem Soc. 87 (1965) 3340.

[2] Hoffmann, R.; Chen, M. L.; Thorn, D. L.: Qualitative discussion of alternative coordination modes of diatomic ligands in transition metal complexes. Inorg. Chem 16 (1977) 503.

[3] Ishikawa, T.; Tanaka, K.: Theoretical study of the photinduced transfer among the ground state and two metastable states in $\left[\mathrm{Fe}(\mathrm{CN})_{5} \mathrm{NO}\right]^{2-}$. J. Chem. Phys. 122 (2005) 074314.

[4] Delley, B.; Schefer, J.; Woike, T.: Giant lifetimes of optically excited states and the elusive structure of sodiumnitroprusside. J. Chem. Phys. 107 (1997) 10067. 
[5] Pressprich, M. R.; White, M. A.; Vekhter, Y.; Coppens, P.: Aanalysis ov a metastable electronic excited state of sodium nitroprusside by X-ray crystallography. J. Am. Chem Soc. 116 (1994) 5233.

[6] Carducci, M. D.; Pressprich, M. R.; Coppens, P.: Diffraction studies of photoexcited crystals: Metastable nitrosyl-linkage isomers of sodium nitroprusside. J. Am. Chem Soc. 119 (1997) 2669.

[7] Gorelsky, S. I.; Lever, A. B. P.: Metastable staates of ruthenium (II) nitrosyl complexes and comparison with $\left[\mathrm{Fe}(\mathrm{CN})_{5} \mathrm{NO}\right]^{2-}$. Int. J. Quant. Chem. 80 (2000) 636.

[8] Blaha, P.; Schwarz, K.; Faber, W.; Luitz, J.: Calculations of electric field gradients in solids: how theory can complement experiment. Hyp. Int. 126 (2000) 389.

[9] Boulet, P.; Buchs, M.; Chermette, H.; Daul, C.; Gilardoni, F.; Rogemond, F.; Schlapfer, C. W.; Weber, J.: Dft investigation of metal complexes containing a nitrosyl ligand. J. Phys. Chem A105 (2001) 8991 and Boulet, P.; Buchs, M.; Chermette, H.; Daul, C.; Furet, E.; Gilardoni, F.; Rogemond, F.; Schlapfer, C. W.; Weber, J.; ibid 8999.

[10] Coppens, P.; Novozhilova, I.; Kovalevsky, A.: Photoinduced linkage isomers of transition-metal nitrosyl compounds and related complexes. Chem. Rev. 102 (2002) 861.

[11] Schaniel, D.; Schefer, J.; Delley, B.; Imlau, M.; Woike, T.: Lightinduced absorption changes by excitatio of metastable states in $\mathrm{Na}_{2}\left[\mathrm{Fe}(\mathrm{CN})_{5} \mathrm{NO}\right] \cdot 2 \mathrm{H}_{2} \mathrm{O}$ single crystals. Phys. Rev. B66 (2002) 085103 .

[12] Schaniel, D.; Woike, T.; Delley, B.; Boskovic, C.; Biner, D.; Kramer, K. W.; Güdel, H.-U.: Long-lived light-induced metastable states in trans- $\left[\mathrm{Ru}\left(\mathrm{NH}_{3}\right)_{4}\left(\mathrm{H}_{2} \mathrm{O}\right) \mathrm{NO}_{2} \mathrm{Cl}_{3} \mathrm{H}_{2} \mathrm{O}\right.$ and related compounds. Phys. Chem. Chem. Phys. 7 (2005) 1164.

[13] Schaniel, D.; Woike, T.; Delley, B.; Schefer, J.; Imlau, M.: Comment on 'Theoretical study of the photoinduced transfer among the ground state and two metastable states in $\left[\mathrm{Fe}(\mathrm{CN})_{5} \mathrm{NO}^{2-}\right.$. J. Chem. Phys. 123 (2005) 047101.

[14] Schaniel, D.; Cormary, B.; Malfant, I.; Woike, T.; Delley, B.; Krämer, K. W.; Güdel, H.-U.: Photogeneration of two metastable NO linkage isomers with high populations of up to $76 \%$ in trans-[RuCl(py) $\left.)_{4}(\mathrm{NO})\right][\mathrm{PF} 6]_{2} \cdot 1 / 2 \mathrm{H}_{2} \mathrm{O}$. Phys. Chem. Chem. Phys. 9 (2007) 3717.
[15] Delley, B.: An All-Electron Numerical Method for Solving the Local Density Functional for Polyatomic Molecules. J. Chem. Phys. 92 (1990) 508.

[16] Delley, B.: From molecules to solids with the $\mathrm{DMol}^{3}$ approach. J. Chem. Phys. 113 (2000) 7756.

[17] Delley, B.: The conductor-like screening model for polymers and surfaces. Molecular Simulation 32 (2006) 117-123.

[18] Govind, N.; Petersen, M.; Fitzgerald, G.; King-Smith, D.; Andzelm, J.: A generalized synchronous transit method for transition state location. Comp. Mat. Sci. 28 (2003) 250.

[19] Perdew, J. P.; Burke, K.; Ernzerhof, M.: Generalized Gradient Approximation Made Simple. Phys. Rev. Lett. 77 (1996) 3865.

[20] Delley, B.: Ground-State Enthalpies: Evaluation of Electronic Structure Approaches with Emphasis on the Density Functional Method. J. Phys. Chem. A110 (2006) 13632.

[21] Schaniel, D.; Woike, T.; Tsankov, L.; Imlau, M.: Evidence of four light-induced metastable states in iron-nitrosyl complexes. Thermochimica Acta 429 (2005) 19-23.

[22] Schaniel, D.; Woike, T.; Schefer, J.; Petricek, V.: Structure of the light-induced metastable state SII in $\mathrm{Na}_{2}\left[\mathrm{Fe}(\mathrm{CN})_{5} \mathrm{NO}\right]$ . $2 \mathrm{H}_{2}$ O. Phys. Rev. B71 (2005) 174112.

[23] Schefer, J.; Woike, T.; Imlau, M.; Delley, B.: Possible reaction coordinates in the metastable states of sodiumnitroprusside $\mathrm{Na}_{2}\left[\mathrm{Fe}(\mathrm{CN})_{5} \mathrm{NO}_{3} \mathrm{H}_{2} \mathrm{O}\right.$. Eur. Phys. J. B3 (1998) 349.

[24] Guida, J. A.; Piro, O. E.; Aymonino, P. J.: Polarized Infrared-Absorption Spectra of $\mathrm{Na}_{2}\left[\mathrm{Fe}(\mathrm{CN})_{5} \mathrm{NO}\right] \cdot 2 \mathrm{H}_{2} \mathrm{O}$ with Part of the Anions in the Electronically Excited Metastable State. Sol. State Comm. 57 (1986) 175.

[25] Krasser, W.; Woike, T.; Haussühl, S.; Kuhl, J.; Breitschwerdt, A.: Resonance Raman-Scattering from the Metastable Electronic State of an $\mathrm{Na}_{2}\left[\mathrm{Fe}(\mathrm{CN})_{5} \mathrm{NO}\right] \cdot 2 \mathrm{H}_{2} \mathrm{O}$ Single-Crystal. J. Ram. Spect. 17 (1986) 83-87.

[26] Baker, J.; Muir, M.; Andzelm, J.: Study of some Organic-Reactions Using Density-Functional Theory. J. Chem. Phys. 102 (1995) 2063-2079.

[27] Zhao, Y.; Truhlar, D. G.: Design of density functionals that are broadly accurate for thermochemistry, thermochemical kinetics, and nonbonded interactions. J. Phys. Chem. A109 (2005) 5656-5667. 